\title{
A Quasi-Brittle damage model in the framework of Bond-based Peridynamics with Adaptive Dynamic Relaxation method
}

\author{
H. N. Yakin, N. Nikabdullah, M. R. M. Rejab \\ Faculty of Mechanical and Automotive Engineering Technology, Universiti Malaysia Pahang, 26600 Pekan, Pahang, Malaysia \\ Phone: +6094246234; Fax: +609424222
}

ABSTRACT - Peridynamics (PD) is a new tool, based on the non-local theory for modelling fracture mechanics, where particles connected through physical interaction used to represent a domain. By using the PD theory, damage or crack in a material domain can be shown in much practical representation. This study compares between Prototype Microelastic Brittle (PMB) damage model and a new Quasi-Brittle (QBR) damage model in the framework of the Bond-based Peridynamics (BBPD) in terms of the damage plot. An in-house code using Matlab was developed for BBPD with inclusion of both damage models, and tested for a quasi-static problem with the implementation of Adaptive Dynamic Relaxation (ADR) method in the theory in order to get a faster steady state solutions. This paper is the first attempt to include ADR method in the framework of BBPD for QBR damage model. This paper analysed a numerical problem with the absence of failure and compared the displacement with literature result that used Finite Element Method (FEM). The obtained numerical results are in good agreement with the result from FEM. The same problem was used with the allowance of the failure to happen for both of the damage models; PMB and QBR, to observe the damage pattern between these two damage models. PMB damage model produced damage value of roughly twice compared to the damage value from QBR damage model. It is found that the QBR damage model with ADR under quasi-static loading significantly improves the prediction of the progressive failure process, and managed to model a more realistic damage model with respect to the PMB damage model.
ARTICLE HISTORY

Received: $18^{\text {th }}$ Jan. 2021

Revised: $16^{\text {th }}$ Nov. 2021

Accepted: 22 ${ }^{\text {nd }}$ Nov. 2021

\section{KEYWORDS}

Peridynamics;

bond-based;

fracture mechanics;

dynamic relaxation.

\section{INTRODUCTION}

Fracture mechanics is the specialisation field in solid mechanics that deal with the study of the propagation of cracks in materials. It lies under continuum mechanics where it relates to the physical study of continuous materials in preference to discrete particles. The continuum assumption states that it models a material by a continuous mass rather than discrete particles [1]. Classical mechanics dealing with the set of physical concept characterise the interaction of bodies and forces. It was developed with the study of the relationship between mass, force, and acceleration. Peridynamics (PD) was introduced in Silling [2] and Silling et. al. [3] as a nonlocal theory with an aim to deal with the discontinuities. The original PD formulation developed by Silling [2], was later called the BBPD theory. The aim of this work is to study damage plot for two different bond damage models namely Prototype Micro-elastic Brittle (PMB) and Quasi-brittle (QBR) using the framework of Bond-based Peridynamics with Adaptive Dynamic Relaxation (ADR).

\section{BASICS OF PERIDYNAMICS THEORY}

The idea of PD was implemented by Silling [3] as a non-local continuum theory to be used as another approach on dealing with failure in material. PD is a numerical method in which particles' interaction occurs over a certain prescribed distance. Easily being said, the main purpose of PD is that it is able to reformulate the solid mechanics equation of motion and the advantages of it are obvious as it uses a spatial integral equation and does not require spatial derivatives to be applied at discontinuities. In PD theory, a number of discrete particles, $\mathbf{x}$, represent a body, interact with surrounding particles in a local region called the horizon, $\delta$. The interactions happen through an inter-particle force functions called PD forces. For a continuous body, an infinite number of connections exist between particle $\mathbf{x}$ and the others in its horizon. The force between two interacting particles can be demonstrated through nonlocal interaction within a continuous body. The particles interactions were based on the deformation of the particles that connected through micropotential. The forces on particle are calculated using integration and when any cracks or failure appear in the body this basic equation can be employed. By using the theoretical background of classical continuum mechanics, the equation of motion is obtained from the conservation of linear momentum, shown as:

$$
\rho(\mathbf{x}) \ddot{\mathbf{u}}(\mathbf{x}, t)=\nabla \cdot \boldsymbol{\sigma}+\mathbf{b}(\mathbf{x}, t)
$$


where $\rho$ is the value of mass density in the undeformed configuration, $\ddot{\mathbf{u}}$ is the second-order derivatives of displacement $\mathbf{u}, \boldsymbol{\sigma}$ is the Cauchy stress whereas $\mathbf{b}$ is prescribed body force density field. Neverthless, the spatial derivative in Eq. (1) cannot be defined along discontinuities . Contrastingly, PD utilise integral function of a force on particle at $\mathbf{x}$ to replace the divergence of stress term, that is [4]

$$
\rho(\mathbf{x}) \ddot{\mathbf{u}}(\mathbf{x}, t)=\int_{R_{x}} \mathbf{f}(\eta, \xi) d V_{\mathbf{x}^{\prime}}+\mathbf{b}(\mathbf{x}, t),
$$

in which $\mathbf{f}$ is the pairwise PD force function that the particle $\mathbf{x}^{\prime}$ applies on $\mathbf{x}, d V_{\mathbf{x}}$ ' is volume associated with particle $\mathbf{x}^{\prime}$ and $\mathrm{R}$ is a neighbourhood of $\mathbf{x}$ and the interaction between particles can be called the bond. The relative position of two particles represented by $\xi$ and relative displacement by $\eta$.

and

$$
\xi=\mathbf{x}^{\prime}-\mathbf{x}
$$

$$
\eta=\mathbf{u}\left(\mathbf{x}^{\prime}, t\right)-\mathbf{u}(\mathbf{x}, t)
$$

\section{Bond-based PD Method}

The BBPD is the original version of PD theory developed by Silling [2], where the interactions between particles act like spring forces. The interactions only rely on the relative displacement of the interacting particles pair and are unrelated to all other local conditions. This shows that the connection of two particles are insensitive to the states of other particles. For a BBPD, the equation of motion in the reference configuration at time $t$ is [2]

$$
\rho \ddot{\mathbf{u}}(\mathbf{x}, t)=\int_{R} \mathbf{f}\left[\mathbf{u}\left(\mathbf{x}^{\prime}, t\right)-\mathbf{u}(\mathbf{x}, t), \mathbf{x}^{\prime}-\mathbf{x}\right] d V_{\mathbf{x}^{\prime}}+\mathbf{b}(\mathbf{x}, t), \quad \forall \mathbf{x}^{\prime} \in R .
$$

The vectors $\mathbf{x}$ and $\mathbf{x}$ ' are defined by the equation:

$$
\mathbf{x}=\left(\begin{array}{c}
x \\
y
\end{array}\right)_{\text {and }} \quad \mathbf{x}^{\prime}=\left(\begin{array}{c}
x^{\prime} \\
y^{\prime}
\end{array}\right)
$$

A scalar $\delta$ is assumed for each material to determine the interacting range between the particle such that,

$$
\mathbf{f}(\eta, \xi)=0 \quad \text { if } \quad\|\xi\|>\delta
$$

Based on the Newton's Third Law, the force function in Eq. (5) must fullfill the linear admissibility condition. Newton's Third Law states that the force at particle $\mathbf{x}$ due to a particle at $\mathbf{x}$ ' must be equal the negative of the force at particle $\mathbf{x}^{\prime}$ due to a particle at $\mathbf{x}$. Therefore for Eq. (5) to satisfy Newton's Third Law, the force function must satisfy:

$$
\mathbf{f}(-\eta,-\xi)=-\mathbf{f}(\eta, \xi)
$$

which assures conservation of linear momentum and admissibility condition which assures conservation of angular momentum. With the conservation of angular momentum, the particles will only move with the presence of external forces, satisfy

$$
(\eta+\xi) \times \mathbf{f}(\eta, \xi)=0
$$

where " $x$ " is the cross product, indicate that the force between any two particles must be parallel to their relative deformation.

\section{METHODOLOGY}

\section{Quasi-Brittle (QBR) Damage Model}

A bond damage model was implemented in [5] based on the BBPD theory to express the progressive failure process in cohesive brittle materials in a better way. This model has been named as QBR model that stand for Quasi-BRittle. The main contribution of the model is to describe the mechanical reaction in between the bonds for cohesive quasi-brittle materials. The formulation is as below:

$$
f= \begin{cases}c s, & s<s_{0} \\ c s_{0}\left[\exp \left(-k \frac{s-s_{0}}{s_{0}}\right)+\alpha \frac{s-s_{0}}{s}\right], & s>s_{0} .\end{cases}
$$

By using this model, the bond status coefficient, $\mu$ can be evaluated as: 
which can be rewritten as:

$$
\mu(\boldsymbol{\xi})= \begin{cases}1, & s \leq s_{0} \\ \frac{f}{c s_{0}}, & s>s_{0}\end{cases}
$$

$$
\mu(\boldsymbol{\xi})= \begin{cases}1, & s \leq s_{0} \\ \exp \left(-k \frac{s-s_{0}}{s_{0}}\right)+\alpha \frac{s-s_{0}}{s}, & s>s_{0} .\end{cases}
$$

In the classical bond damage model called Prototype Micro-elastic Brittle (PMB), the bond force value increases linearly with the bond stretch but when the stretch value reaches its critical value, the bond force abruptly drops to zero. Eq. (10) shows the evaluation of the mechanical response or force of bonds for cohesive quasi-brittle materials. In this Tong's proposed model [5], $k$ is the parameter that represent the reduction of the bond force and $\alpha$ controls the residual bond force, $s$ is the bond stretch while $s_{0}$ represents the elastic limit of bond stretch at the peak force, and $c$ is the local bond modulus. The classical model is widely used for modelling fracture problems in linear elastic brittle materials but it is not suit for most cohesive brittle materials since the failure is generally a progressive process. The exponential law was found to be the most consistent with the experimental results [5]. The macroscopic damage state variable can be evaluated as

$$
\varphi(\boldsymbol{x}, t)=1-\frac{\int_{H} \mu(\xi, t) d V_{\xi}}{\int_{H} d V_{\xi}}
$$

which $\varphi=0$ bring the meaning of undamaged state and $\varphi=1$ means that the material point $\mathrm{x}$ is completely in damage.

\section{Adaptive Dynamic Relaxation (ADR) Method}

Since the equations of PD utilise explicit time integration, when it comes to a problem that is under quasi-static loading conditions, it is troublesome in analysing it. Kilic and Madenci proposed an additional formulation in dynamic relaxation methods to achieve steady-state solutions in the framework of nonlinear PD equations [6]. This method originated from dynamic relaxation method by instigating an unnatural damping to be included in the system but still determining the most effective damping coefficient is not always achievable. Papadrakakis [7] and Underwood [8] proposed the adaptive dynamic relaxation where in this method the changes of damping coefficient is happens adaptively for every iteration step. The equation can be include into set of ordinary differential equations that is appropriate with the theory of dynamic relaxation. The acceleration term is taken out from the equation. Instead it introduced a fictitious diagonal density and damping matrix that is proportional to the density matrix as

$$
\Lambda \ddot{\mathbf{U}}(\mathbf{X}, t)+c \Lambda \dot{\mathbf{U}}(\mathbf{X}, t)=\mathbf{F}\left(\mathbf{U}, \mathbf{U}^{\prime}, \mathbf{X}, \mathbf{X}^{\prime}\right)
$$

where $\Lambda$ represent the fictitious diagonal density matrix and $c$ exhibit the damping coefficient that is determine by Greschgorin's theorem [8]. $\mathbf{X}$ is initial position of the particles, and $\mathbf{U}$ is displacement of the particles. Both of the parameter can be denote as [8]

and

$$
\mathbf{X}^{T}=\left\{\mathbf{x}, \mathbf{x}_{2}, \ldots, \mathbf{x}_{N}\right\}
$$

$$
\mathbf{U}^{T}=\left\{\mathbf{u}\left(\mathbf{x}_{1}, t\right), \mathbf{u}\left(\mathbf{x}_{2}, t\right), \ldots, \mathbf{u}\left(\mathbf{x}_{N}, t\right)\right\}
$$

$N$ stands for the total number of particles. By employing central-difference explicit integration, velocities and displacement for the next iteration can be evaluated by:

and

$$
\dot{\mathbf{U}}^{n+1 / 2}=\left[(2-c \Delta t) \dot{\mathbf{U}}^{n-1 / 2}+2 \Delta t \Lambda^{-1} \mathbf{F}^{n}\right] /[2+c \Delta t],
$$

$$
\mathbf{U}^{n+1}=\mathbf{U}^{n}+\Delta t \dot{\mathbf{U}}^{n+1 / 2}
$$

where $n$ represents the $n$th iteration, $\Delta t$ is the time step size. The best time step size in dynamic relaxation is $1,(\Delta t=1)$ [6]. The above expression cannot be employed at the beginning of the integration because the velocity field at $t^{-1 / 2}$ is unknown, but the process can be commenced by using:

$$
\dot{\mathbf{U}}^{1 / 2}=\Delta t \boldsymbol{\Lambda}^{-1} \mathbf{F}^{0} / 2 \text {. }
$$


There is no need for the density matrix, $\boldsymbol{\Lambda}$, damping coefficient, $c$ and time step size, $\Delta t$ to be physically meaningful quantities [6]. It means to obtain faster convergence the value can be chosen. The most common method to choose density matrix is based on Gerschgorin's theorem, which can be written as [8]:

$$
\Lambda^{-1}=\frac{1}{4} \Delta t^{2} \sum_{j}\left|k_{i j}\right|,
$$

where $i$ is the corresponding particle and $j$ is the particle connected to the corresponding particle $i$ and $k_{i j}$ calculated as [9]

$$
k_{i j}=5 \pi \delta^{2} b c
$$

in which $b c$ is the bond constant expressed as:

$$
b c=\frac{18 K}{\pi \delta^{4}},
$$

where $K$ indicates the bulk modulus and $\delta$ is the radius of PD horizon. The damping coefficient $c$ expressed as:

$$
c^{n}=2 \sqrt{\left(\left(\mathbf{U}^{n}\right)^{T} \mathbf{K}^{n} \mathbf{U}^{n}\right) /\left(\left(\mathbf{U}^{n}\right)^{T} \mathbf{U}^{n}\right)},
$$

$\mathbf{K}^{n}$ represents the diagonal local stiffness matrix, which is given as:

$$
K_{i i}^{n}=-\left(\mathbf{F}_{i}^{n} / \boldsymbol{\Lambda}^{-1}-\mathbf{F}_{i}^{n-1} / \boldsymbol{\Lambda}^{-1}\right) /\left(\Delta t \dot{\mathbf{U}}_{i}^{n-1 / 2}\right) .
$$

\section{NUMERICAL EXAMPLE}

An isotropic plate with a hole at the centre under quasi-static loading along its horizontal edges was analysed. In this problem, there is no existing crack in its domain. The geometric parameters of the plate are as stated below; both length, $l$ and width, $w$ of $50 \mathrm{~mm}$, the plate's thickness, $h$ of $0.5 \mathrm{~mm}$, and diameter of the circular hole in the middle of specimen, $D$ of $10 \mathrm{~mm}$. The material properties of the plate are as stated here with Young's modulus, $E$ of $192 \mathrm{GPa}$, Poisson's ratio, $v$ of $1 / 3$, and mass density, $\rho$ of $8000 \mathrm{~kg} / \mathrm{m}^{3}$. The boundary condition applied to the plate by applying velocity, $v$ of $\pm 2.7541 \times 10^{-7} \mathrm{~m} / \mathrm{s}$. The number of particle discretisation in $\mathrm{x}$-direction is 100 particles, and the number of particle discretisation in the $y$-direction is $100+3+3$, whereas in the z-direction is 1 . Particles spacing, $\Delta \mathbf{x}$ is taken to be 0.0005 $\mathrm{m}$. The horizon, $\delta$ is equal to $3.015 \Delta \mathbf{x}$. The critical stretch value, $s_{0}$ is taken to be 1 when the failure is not allowed, and the $s_{0}$ value is 0.04472 when the failure is allowed to happen.

The displacement of the specimen was plotted together with the results obtained from finite element analysis from the literature [9]. The failures are not allowed to happened in this test by prescribing the value of stretch limit $s_{0}$ to a big value such as 1. Figure 1(a) showed the horizontal displacement plot across the length of middle x-axis. Whereas in Figure 1(b) it showed the vertical displacement plot across the length of the central y-axis. From both of these plots, it can be seen that both plot from PD and FEM have close consistency [9]. It can be concluded that the accurate results from PD analysis can be achieved by setting up the correct parameter value for the size of discretisation, horizon size, and the volume of the region. All the parameter values in PD analysis is used for the next analysis where the failure in the body will be allowed to happen by specifying the critical stretch value, $s_{0}$ to be equal to 0.04472 . The damage initiation and development is explored at different time steps. It is to be noted that there are no pre-existing crack exist in the plate, but still the method can shows that the failure initiates as a crack at high stress concentration location. This is a truly exceptional advantage of using PD analysis. The initiation of damage at the stress concentration location begins in the time steps 669. Figure 2(a) shows the damage plot using PMB damage model at time steps of 700, 800, 900 and 1000. Figure $2 \mathrm{~b}$ showed that some of the particles in the domain experienced the damage value of more than 0.4 , that will results in self-similar crack branching. Since the loading applied in quasi-static loading; where the value of applied velocity on the edge of the body is very slow, the propagation of the cracks move toward the outer side of vertical boundaries, as shown in Figures 2(c) and 2(d).

The analysis is then continued using the QBR damage model that was introduced in [5]. In this model, the connection or bond between two particles do not break straight away when the value of stretch exceed the stretch limit. However, the force value will drops exponentially before the bond is completely breaks. This kind of damage model is more realistic especially when modelling brittle materials that will experience micro-cracks throughout the loading process [5]. As it can be seen from Figures 3(b) and 2(b), the damage value for QBR is lower than the damage value from the PMB. This means that the QBR failure in the bonds do not happen abruptly as in the PMB model, and the bond can still sustain the stretch even after it reaches the stretch limit value, $s_{0}$. Besides, from Figures 2(d) and 3(d), it can be seen that the damage variation contour in QBR damage model is more realistic, not as in the PMB damage model where it shows a non-uniform pattern along the damage plot [10]. In particular, the new bond damage model significantly improves the prediction of the post-peak responses with respect to the classical elastic- brittle bond model [9]. 

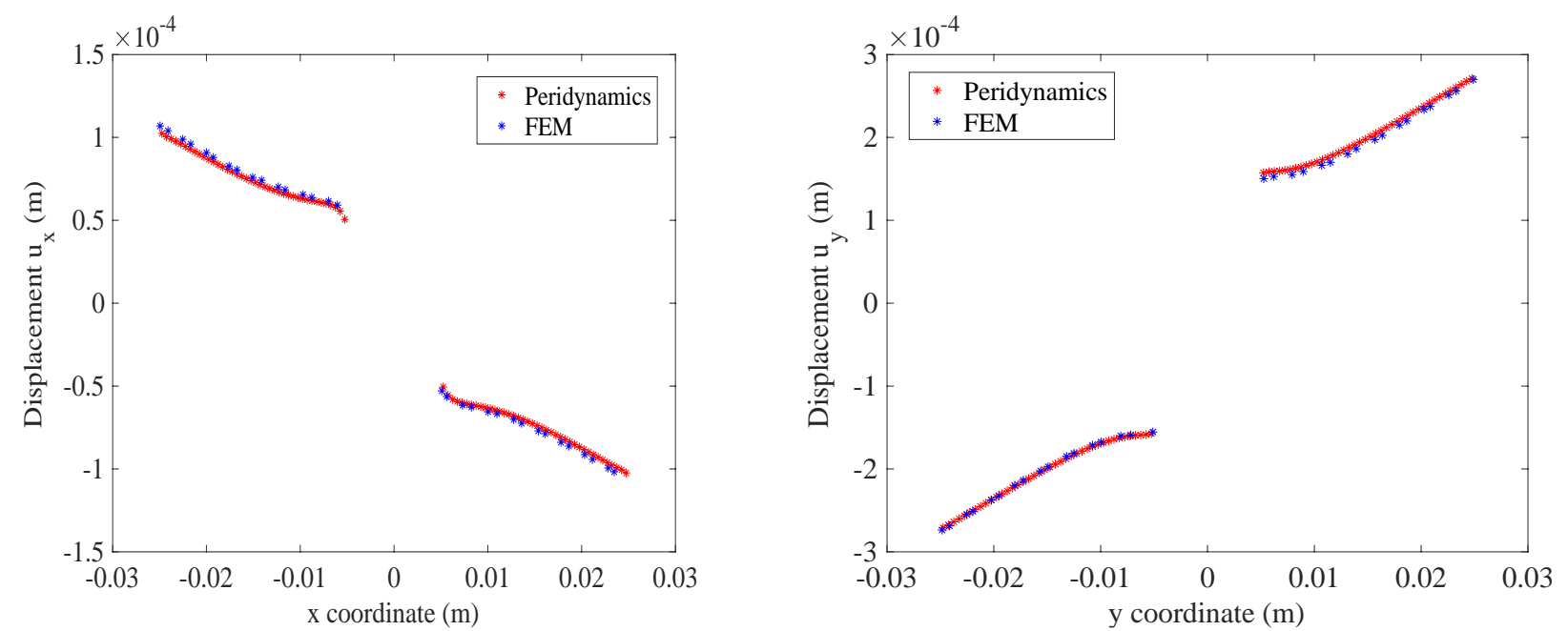

Figure 1. The plot of: (a) horizontal displacement, and (b) vertical displacement, across the central axes at the end of 1,000 time steps when failure is absence

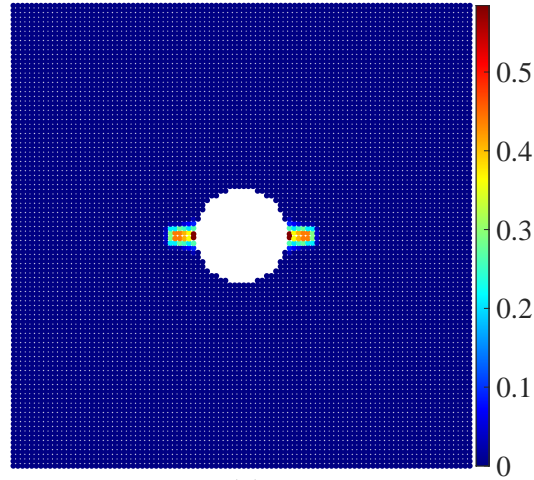

(a)

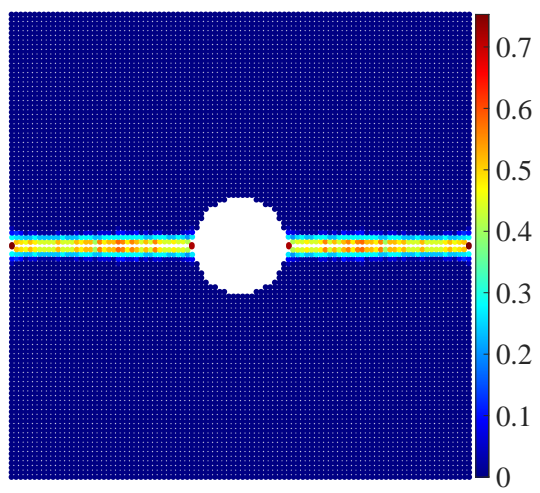

(c)

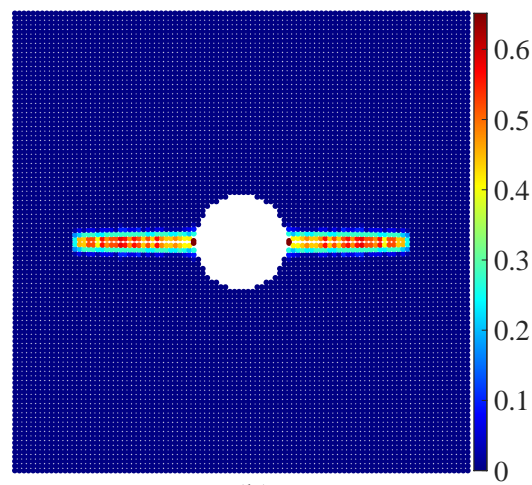

(b)

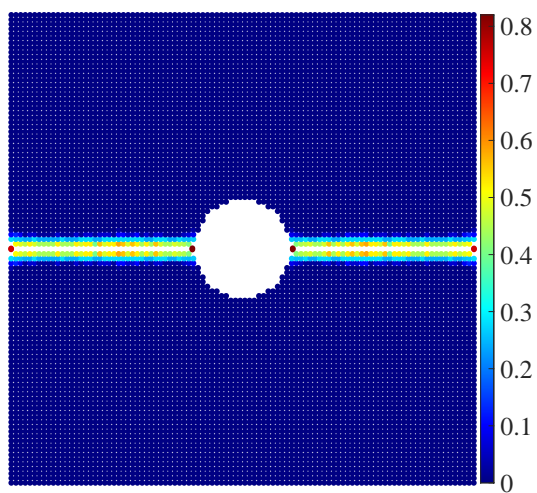

(d)

Figure 2. Damage plot using PMB damage model for time steps: (a) 700, (b) 800, (c) 900 and (d) 1000 


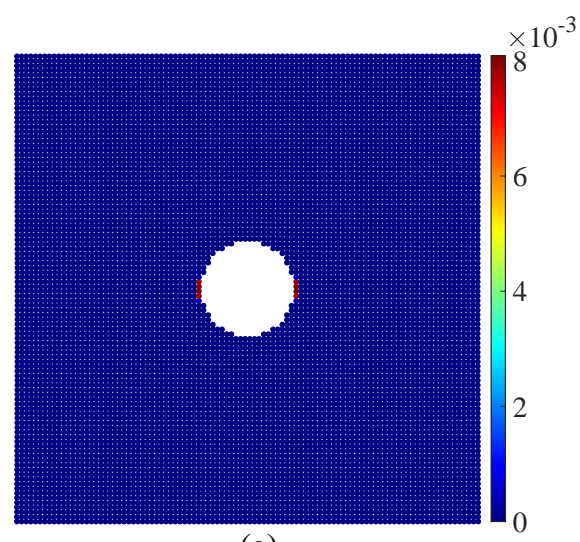

(a)

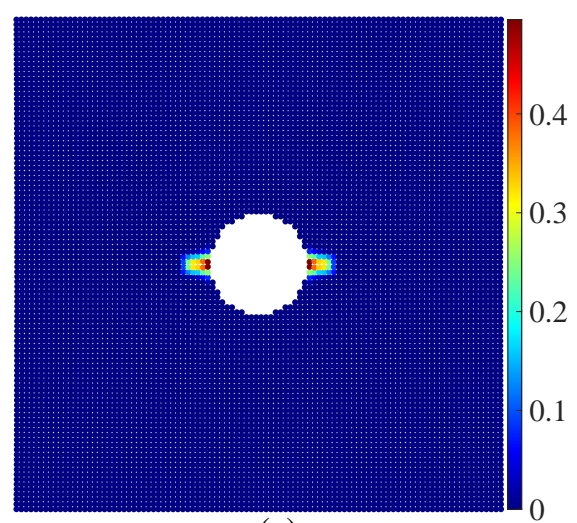

(c)

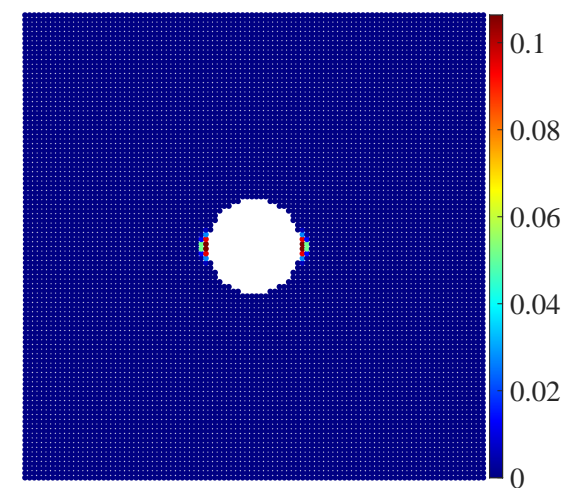

(b)

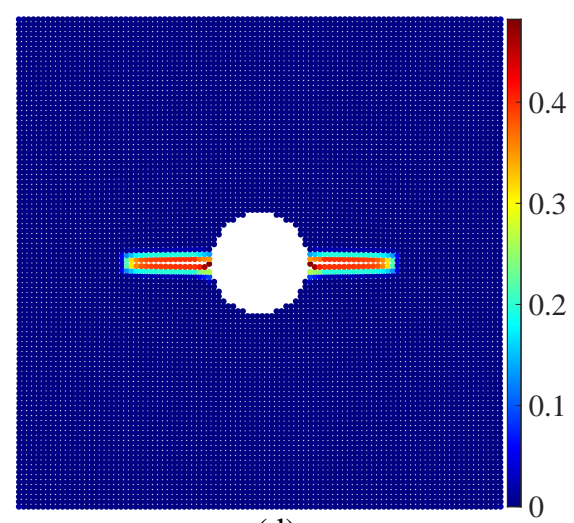

(d)

Figure 3. Damage plot using QBR damage model for time steps: (a) 700, (b) 800, (c) 900 and (d) 1000 damage model

\section{CONCLUSION}

In this paper, a comparison between PMB damage model and a new QBR damage model in the framework of the BBPD in terms of damage plot has been presented. An in-house code for BBPD with inclusion of the both damage model has been developed. A quasi-static problem has been tested where the ADR method has been implemented in the simulation in order to get a faster steady state solutions. First, a numerical problem with the absence of failure has been analysed and the displacements were compared with result from FEM [9]. The obtained numerical results showed good agreement with the result from FEM. The same problem has been used by allowing the failure to happen for both of the damage model; PMB and QBR. The damage pattern between these two damage models has been observed. PMB damage model produced damage value of roughly twice compared to the damage value from QBR damage model. It is found that the damage model with ADR under quasi-static loading significantly improves the prediction of failure process by taking into account the proggressive degradation of PD's bond stiffness. This damage model managed to model a more realistic damage model with respect to the PMB damage model. This model therefore opens the door for PD to be used for a wider class of material in the prediction of failure.

\section{ACKNOWLEDGEMENT}

The authors would like to thank the Ministry of Higher Education for providing financial support under Fundamental Research Grant Scheme (FRGS) No. FRGS/1/2017/TK05/UMP/01/1 (University reference RDU170131) and Universiti Malaysia Pahang for laboratory facilities as well as additional financial support under Internal Research grant PGRS2003140.

\section{REFERENCES}

[1] G. T. Mase, R. E. Smelser, and G. E. Mase, Continuum Mechanics for Engineers, Third Edition. CRC Press, 2009.

[2] S. A. Silling, "Reformulation of elasticity theory for discontinuities and long-range forces," J. Mech. Phys. Solids, vol. 48, no. 1, pp. 175-209, 2000, doi: 10.1016/S0022-5096(99)00029-0.

[3] S. A. Silling, M. Epton, O. Weckner, J. Xu, and E. Askari, "Peridynamic States and Constitutive Modeling," J. Elast., vol. 88, no. 2, pp. 151-184, 2007, doi: 10.1007/s10659-007-9125-1. 
[4] M. L. Parks, R. B. Lehoucq, S. J. Plimpton, and S. A. Silling, "Implementing peridynamics within a molecular dynamics code," Comput. Phys. Commun., vol. 179, no. 11, pp. 777-783, 2008, doi: 10.1016/j.cpc.2008.06.011.

[5] Y. Tong, W. Shen, J. Shao, and J. Chen, "A new bond model in peridynamics theory for progressive failure in cohesive brittle materials," Eng. Fract. Mech., vol. 223, no. October 2019, p. 106767, 2020, doi: 10.1016/j.engfracmech.2019.106767.

[6] B. Kilic and E. Madenci, "An adaptive dynamic relaxation method for quasi-static simulations using the peridynamic theory," Theor. Appl. Fract. Mech., vol. 53, no. 3, pp. 194-204, 2010, doi: 10.1016/j.tafmec.2010.08.001.

[7] M. Papadrakakis, "A method for the automatic evaluation of the dynamic relaxation parameters," Comput. Methods Appl. Mech. Eng., vol. 25, no. 1, pp. 35-48, 1981, doi: 10.1016/0045-7825(81)90066-9.

[8] P. Underwood, “Computational Methods for Transient Analysis 1,” in Dynamic Relaxation, 1983, pp. $245-265$.

[9] E. Madenci and E. Oterkus, Peridynamic Theory and Its Applications, vol. c, no. 2. New York, NY: Springer New York, 2014.

[10] W. Gerstle, N. Sau, and S. A. Silling, "Peridynamic modeling of concrete structures," Nucl. Eng. Des., vol. 237, no. 12-13, pp. 1250-1258, 2007, doi: 10.1016/j.nucengdes.2006.10.002. 